\title{
Evaluation of a pharmaceutical assessment screening tool to measure patient acuity and prioritise pharmaceutical care in a UK hospital
}

\author{
Ryan P Hickson, ${ }^{1}$ Douglas T Steinke, ${ }^{2,3}$ Charlotte Skitterall, ${ }^{3}$ Steven D Williams ${ }^{2,3}$
}

'Division of Pharmaceutical Outcomes and Policy, Eshelman School of Pharmacy, University of North Carolina at Chapel Hill, Chapel Hill, North Carolina, USA

2Manchester Pharmacy School, University of Manchester, Manchester, UK

${ }^{3}$ University Hospital of South Manchester NHS Foundation Trust, Manchester, UK

\section{Correspondence to} Steven D Williams, The University of Manchester, Stopford Building, Oxford Road, Manchester M13 9PT, UK;

s.williams@manchester.ac.uk

Received 26 October 2015 Revised 19 April 2016 Accepted 28 April 2016 Published Online First 31 May 2016

CrossMark

To cite: Hickson RP, Steinke DT, Skitterall C, et al. Eur J Hosp Pharm 2017:24:74-79.

\section{ABSTRACT}

Objective A service evaluation project was conducted to design a pharmaceutical assessment screening tool (PAST) to assign all inpatients a patient acuity level (PAL) to then help teams of clinical pharmacists prioritise the frequency of, and the seniority of, pharmacists performing patient reviews; assess clinical pharmacists' adherence to the tool; and identify when pharmacists do not adhere to the tool.

Methods The PAST was developed by consensus methodology to prioritise departmental workflow for clinical pharmacists. The most pharmaceutically complex patients at the greatest risk of adverse drug events were expected to receive a PAL score of 3 , while the least complex receive a PAL of 1 . A quasi-experimental service evaluation study was conducted 6 months after implementation of the tool to quantify agreement between pharmacist-documented and expected per-guidance PALs. Patients were selected via random clusters from wards. For each patient, a PAL was calculated by the researcher and compared with the pharmacist-documented PAL.

Results 20 patients (57\%) had documented PALs that matched the expected PAL based on pharmacy departmental guidance. Seven of nine patients with overvalued pharmacist-documented PALs had no high-risk medications and no organ dysfunction. Four of six patients with undervalued pharmacist-documented PALs had cystic fibrosis, who should all automatically score the maximum level.

Conclusions Until electronic health records allow the calculation of PALs automatically, the utilisation of the current tool may be improved by eliminating unclear and unused portions of the tool and reiterating the true purpose of the tool to all pharmacists.

\section{INTRODUCTION}

As UK hospitals increasingly encounter older patients with multiple morbidities and polypharmacy and face demands for a 7-day clinical service, there is an urgent need for pharmacy departments to prioritise which patients need direct pharmaceutical care on a daily basis. Despite the limited evidence that patient outcomes are improved by assessing patient acuity (the ability to predict patient requirements for care), ${ }^{1}$ both nursing and medicine have tools to help assess patient acuity and guide the levels of hospital care and staffing required in the $\mathrm{UK}^{2}{ }^{3}$ Pharmacy practice currently lacks standardised tools to assess patient acuity. However, interest in this area of study has increased in recent years with the goal of assessing patient acuity and the complexity of pharmaceutical regimens to identify patients at the greatest risk of adverse drug events. ${ }^{4-11}$

The American Society of Health-System Pharmacists (ASHP) understands the need to prioritise pharmaceutical care, and in 2013 awarded a $\$ 0.5$ million research grant to develop and validate a pharmaceutical complexity scoring tool. ${ }^{4}$ The tool is designed to improve patient safety and prevent adverse drug events by directing hospital pharmacist care to the patients who should benefit most, in line with the refined US definition of pharmaceutical care. ${ }^{12}$ The complexity score will be measured automatically using the electronic health record to predict, in real time, which patients are at greatest risk of adverse drug events. This tool will however only have utility in a hospital with a fully electronic prescribing and administration system integrated with electronic pathology records.

One hospital in New Zealand has already designed a similar real-time software-based tool to help pharmacists prioritise the prevention of adverse drug events for inpatients. Thirty-eight electronic 'flags' were used to provide an assessment of risk score indicating a low-risk patient, medium-risk patient or high-risk patient. The 38 'flags' were made up from five broad groupings: patient profile (age, ethnicity), patient encounter type (frequency and type of hospital visits), clinical profile (known chronic disease states), high-risk medication (number and type) and laboratory values. The authors report that the scoring tool allowed pharmacists to perform medicines reconciliation and clinical review in a more timely and targeted manner. ${ }^{5}$

Some UK pharmacy departments have developed tools to identify patients at greater risk of experiencing medication errors and adverse drug events. In Scotland, a clinical pharmacy team developed a pharmaceutical care priority screening tool to assign patient-based risk scores using an electronic prescribing and medicines administration (EPMA) system in response to a serious medication error that occurred on a ward without a clinical pharmacy service. ${ }^{6}$ This helped change the model of care from ward based to patient focused. Another UK hospital used a similar EPMA system to develop a web-enabled 'portal' that allowed pharmacists to look at multiple patient characteristics for any given ward and thus help them prioritise which patients needed to be reviewed. ${ }^{7}$ The portal included scores and warnings based on time since admission, medicines reconciliation status, the number of high-risk medicines, pharmaceutical 
problems, including drug-drug interactions and pharmaceuticalbiochemistry alerts such as heparin-induced thrombocytopenia. Pharmacists were surveyed about the impact of the medicationbased risk assessment tool on their clinical practice and scored it highly as a method to help them prioritise patients to be reviewed (mean score 4.9 from ' 1 : Isn't relevant' to '6: Couldn't work without it').

While both these tools used an electronic prescribing system, they did not include patient comorbidities or serum laboratory values. These could both help identify drug-disease interactions and the need for dose adjustment due to renal/hepatic dysfunction, which are important factors for pharmacists wishing to determine patient acuity. Safadeh et $a l^{8}$ also found that a tool assessing patient and medication factors (renal/hepatic function, polypharmacy, adverse drug reactions, therapeutic drug monitoring (TDM), drug administration and medication specific issues) could help identify complex patients admitted to a medical admissions unit which needed referral to a more experienced pharmacist.

The aim of this study was (1) to design a pharmaceutical assessment screening tool (PAST) to assign all inpatients a patient acuity level (PAL) to help guide teams of clinical pharmacists prioritise the frequency of, and the seniority of, pharmacists performing patient reviews; (2) to assess how clinical pharmacists adhere to the PAST by quantifying the level of agreement between the pharmacist-documented PAL and the expected PAL from pharmacy department guidance and (3) to identify any common patterns among patients where the pharmacist-documented PALs do not adhere to the expected PAL.

\section{METHODS}

The study site was a 900-bed teaching hospital in England. A draft of the PAST was originally developed by the consultant pharmacist in medication safety. It was based on similar tools that existed in the literature ${ }^{6-8}$ but included patient-level (adapted from the UK Intensive Care Society's levels of critical care for adult patients ${ }^{2}$ and the Shelford Group's Safer Nursing Care Tool) ${ }^{3}$ and medication-based risk factors based on highrisk medications known to cause serious harm. ${ }^{11}$

A team of senior and junior clinical pharmacists, from different medical and surgical specialties, piloted the use of the tool to confirm face validity. Agreement on the final tool and PALs was then sought by a consensus methodology. The agreed PAST is shown in figure 1. A patient on a high-risk medication or with single organ dysfunction has an expected PAL of 2 per

\section{Level 3 Patients}

- Patient is severely unwell with more than one acutely decompensated organ (kidney, liver, heart, lung, bone marrow, brain)

- Patient is on a high-risk medicine $(\mathrm{HRM})^{*}$ or a medicine requiring Therapeutic drug monitoring (TDM) plus one acutely decompensated organ Patient stepped down from ICU or under review by ICU outreach team

- An acute admission managed (or jointly managed) by an infectious diseases (ID) consultant

- Cystic fibrosis, organ transplant or patients with HIV

- Being considered for home IVs / out-of-hospital respiratory service

- Patient with Parkinson's disease on apomorphine pump

- Patient taking or being considered for a high-cost medicine (eg, posaconazole) that requires alternative funding stream (e.g. Payment by Results $(\mathrm{PbR})$ )

- Patient's condition or drug therapy regimen outside of the competency of a junior pharmacist

Level 2 Patients

- Patient is on a high-risk medicine (HRM)*

- Patient is on a medicine requiring Therapeutic Drug Monitoring (TDM)

- Patient has one acutely decompensated organ

Level 1 Patients

- Low-risk medicines only

- Limited comorbidities

- Medicine regimen stable

- Clinically stable

- Medically fit and awaiting care packages for discharge

*High-Risk Medicines (HRM)

- Anticoagulants warfarin and novel oral anticoagulants (excluding enoxaparin $\leq 40 \mathrm{mg}$ daily)

- Insulin

- Regular strong opiates (excluding codeine/ dihydrocodeine, tramadol)

- Drugs with a Narrow Therapeutic Index

- Chemotherapy

- Antiretrovirals

- Clozapine

Figure 1 Pharmaceutical assessment screening tool (PAST) to assist pharmacists in assigning a patient acuity level (PAL). ICU, intensive care unit; IVs, intravenous medications. 
pharmacy department guidance. A patient has an expected PAL of 3 if they have (a) both a high-risk medication and an organ dysfunction, (b) multiple organ dysfunction or (c) any other factor specified in figure 1 under PAL 3; patients with a PAL of 3 are expected to have the greatest risk of adverse drug events. All other patients are considered to have the lowest level of pharmaceutical complexity and default to PAL 1; these patients are expected to have the lowest risk of adverse drug events.

The PAL for each patient is expected to be recorded when the patient is first seen after admission and then when any changes occur during their hospital admission. The PAL is recorded on the ward's electronic patient summary board, which can be viewed remotely in the pharmacy department. The most experienced pharmacists within a clinical team are then expected to care for the patients with the highest PALs, that is, the most pharmaceutically complex patients at the greatest risk of adverse drug events.

The application of the tool was added to the department's pharmaceutical care guidance in January 2014 and all clinical pharmacists received face-to-face training about the principles, the perceived benefits for work prioritisation within clinical teams and how to calculate PALs using the tool.

To quantify agreement between pharmacist-documented and expected per-guidance PALs, a quasi-experimental service evaluation study was then conducted in July 2014, 6 months after implementation of the tool. Patients were selected via random clusters: wards with adult patients were selected at random followed by random patient selection within these wards. Intensive care units (ICUs) were excluded as all patients in the ICUs were considered PAL 3 patients and automatically received daily pharmacy review from an experienced pharmacist. From each selected ward, five patients were selected at random from those with a pharmacist-documented PAL. Data were collected for 35 patients from seven different wards. For each patient, the research team reviewed handwritten clinical notes, handwritten medication prescriptions and electronic serum laboratory values to determine the PAL according to the departmental PAST guidance. This department-recommended PAL was then compared with the PAL recorded by the pharmacist during normal clinical practice.

Frequencies and percentages were recorded for all factors used within the PAST and sorted by level of PAL agreement. A measure of agreement (values of -2 to +2 ) was calculated by subtracting the expected PAL from the pharmacist-documented PAL. A negative agreement meant the pharmacist undervalued the patient's acuity level; a positive agreement meant the pharmacist overvalued the patient's acuity level. A kappa statistic was calculated to assess agreement between the pharmacistdocumented PALs and the expected PALs recommended by departmental guidance.

\section{RESULTS}

In the evaluation of the tool, all wards had a median pharmacist-documented PAL of level +2 , and five of the seven wards had a median measure of agreement of 0 (figure 2).

The PAST factors for all sampled patients are shown in table 1. The pharmacist-documented PALs had the following distribution: 4 level-1 patients (11\%), 25 level-2 patients (71\%) and 6 level-3 patients (17\%). The distribution of expected PALs was 11 level-1 patients (31\%), 15 level-2 patients (43\%) and 9 level-3 patients $(26 \%)$.

PAL comparisons (pharmacist-documented minus expected PAL) showed that 6 patients (17\%) had a -1 agreement, 20 patients $(57 \%)$ had 0 agreement, 8 patients $(23 \%)$ had a +1 agreement and 1 patient $(3 \%)$ had a +2 agreement. No patients had a -2 agreement.

Further evaluation (table 2) showed that 4 of 11 level-1 patients (36\%), 13 of 15 level-2 patients (87\%) and 3 of 9 level-3 patients (33\%) were in full agreement between the pharmacist-documented and expected level (kappa statistic on agreement, $\kappa=0.344$, suggesting slight agreement). ${ }^{13}$ When a pharmacist documented a patient as Level 1, the PAL was always in agreement with the expected level.

Among the sampled patients, eight had kidney dysfunction and one had liver dysfunction. No patients were found to have any other organ dysfunction and no patients had multiple organ dysfunction. The most common medications requiring TDM were aminoglycosides (four patients) and aminophylline (three patients); however, four of these seven patients (57\%) were patients with cystic fibrosis (CF). Patients with CF are automatically recommended to receive a PAL of 3 and also accounted for six of the nine (67\%) TDM drugs identified. When excluding patients with $\mathrm{CF}$, three patients were on a drug that required TDM (aminoglycoside, glycopeptide and digoxin). Other common high-risk medications were regularly administered opiates, insulin and anticoagulants (including low molecular weight heparins, traditional and novel oral anticoagulants). Eighteen patients (51\%) were on no high-risk medications.

Table 3 shows the PAST factors for all sampled patients sorted by agreement of pharmacist-documented and expected acuity levels. Six patients (four on the CF ward and two with kidney dysfunction) had a PAL agreement of -1 (ie, the pharmacist undervalued the patient's acuity). All of these patients had a pharmacist-documented PAL of 2 and an expected PAL of 3 .
Figure 2 Median patient acuity levels (PALs) for each ward were randomly selected. Wards $A, E, F$ and $G$ are medical wards. Wards $B, C$ and D are surgical wards.

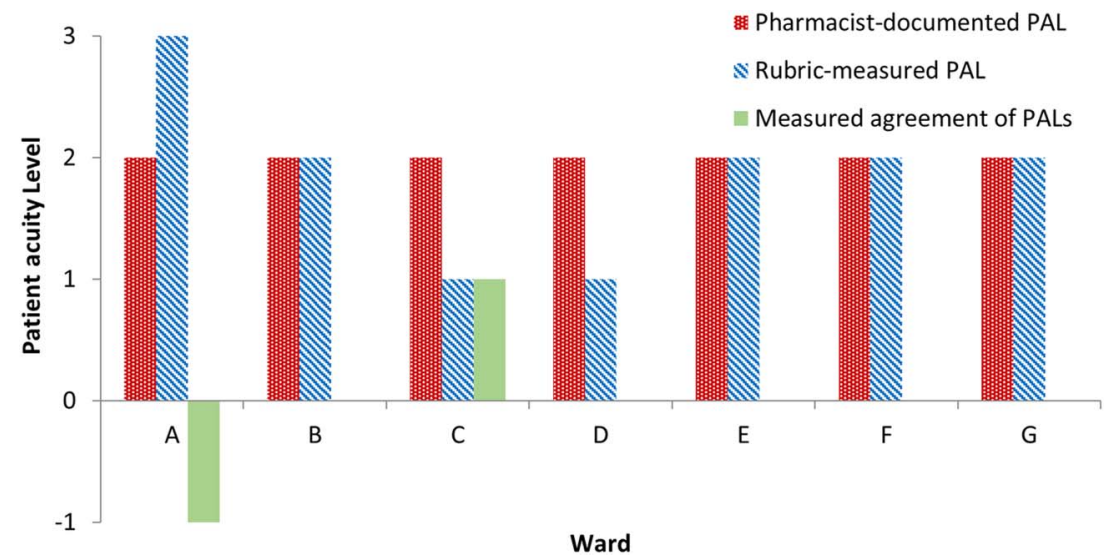

Hickson RP, et al. Eur J Hosp Pharm 2017;24:74-79. doi:10.1136/ejhpharm-2015-000829 
Table 1 Pharmaceutical assessment screening tool (PAST) factors for all sampled patients

\begin{tabular}{|c|c|}
\hline Pharmaceutical assessment screening factors & Number (\%) \\
\hline $\mathrm{N}$ & 35 \\
\hline \multicolumn{2}{|l|}{ Organ dysfunction } \\
\hline Kidney & $8(23)$ \\
\hline Liver & $1(3)$ \\
\hline Heart & $0(0)$ \\
\hline Lung & $0(0)$ \\
\hline Bone marrow & $0(0)$ \\
\hline Brain & $0(0)$ \\
\hline Dysfunction of more than one organ & $0(0)$ \\
\hline \multicolumn{2}{|l|}{ Drugs requiring therapeutic monitoring } \\
\hline Glycopeptide antibiotic & $1(3)$ \\
\hline Aminoglycosides & $4(11)$ \\
\hline Theophylline/aminophylline & $3(9)$ \\
\hline Digoxin & $1(3)$ \\
\hline Carbamazepine & $0(0)$ \\
\hline Lithium & $0(0)$ \\
\hline Ciclosporin & $0(0)$ \\
\hline Amphotericin B & $0(0)$ \\
\hline Triazole antifungals & $0(0)$ \\
\hline \multicolumn{2}{|l|}{ Number of drugs requiring therapeutic monitoring } \\
\hline 0 & $28(80)$ \\
\hline 1 & $5(14)$ \\
\hline 2 & $2(6)$ \\
\hline \multicolumn{2}{|l|}{ Other high-risk medications } \\
\hline Anticoagulation & $5(14)$ \\
\hline Insulin & $8(23)$ \\
\hline Opiates & $6(17)$ \\
\hline Chemotherapy & $0(0)$ \\
\hline Antiretrovirals & $0(0)$ \\
\hline Clozapine & $0(0)$ \\
\hline \multicolumn{2}{|l|}{ Number of high-risk medications* } \\
\hline 0 & $18(51)$ \\
\hline 1 & $8(23)$ \\
\hline 2 & $7(20)$ \\
\hline 3 & $2(6)$ \\
\hline ICU step-down patient & $1(3)$ \\
\hline Patient with an infectious disease consult & $0(0)$ \\
\hline Patient with cystic fibrosis & $5(14)$ \\
\hline Organ transplant patient & $0(0)$ \\
\hline Home intravenous therapy & $0(0)$ \\
\hline Out-of-hospital respiratory service & $0(0)$ \\
\hline Patient with HIV & $0(0)$ \\
\hline Patient with Parkinson's disease on apomorphine & $0(0)$ \\
\hline High-cost medication (not within NHS tariff) & $0(0)$ \\
\hline Outside competency of attending pharmacist $†$ & $0(0)$ \\
\hline \multicolumn{2}{|l|}{ Pharmacist-documented PAL } \\
\hline 1 & $4(11)$ \\
\hline 2 & $25(71)$ \\
\hline 3 & $6(17)$ \\
\hline \multicolumn{2}{|l|}{ Expected PAL } \\
\hline 1 & $11(31)$ \\
\hline 2 & $15(43)$ \\
\hline 3 & $9(26)$ \\
\hline \multicolumn{2}{|l|}{ Measured agreement of PAL $\ddagger$} \\
\hline-2 & $0(0)$ \\
\hline-1 & $6(17)$ \\
\hline 0 & $20(57)$ \\
\hline+1 & $8(23)$ \\
\hline
\end{tabular}

Table 1 Continued

Pharmaceutical assessment screening factors
+2
${ }^{*}$ Number of high-risk medications includes drugs requiring therapeutic monitoring
plus other high-risk medications.
tOutside competency refers to documentation by a junior pharmacist that the
patient's complexity is outside the scope of their competency.
¥Measured agreement of PALs is the difference of pharmacist-documented PAL minus
the expected PAL with a range of values from -2 to +2 .
ICU, intensive care unit; NHS, National Health Service; PAL, patient acuity level.

Eight patients had a PAL agreement of +1 (table 3 ). Six of these eight patients (75\%) had no high-risk medications or organ dysfunction. Of the nine patients with positive PAL agreements (ie, the pharmacist overvalued the patient's acuity), seven (78\%) should have been designated as Level 1 according to the pharmacy department guidance as they did not have any organ dysfunction or high-risk medications.

\section{DISCUSSION}

Twenty patients $(57 \%)$ had a pharmacist-documented PAL that agreed with the expected departmental guidance suggesting that not all pharmacists follow the PAST guidance developed by the pharmacy department. Adherence to clinical practice guidelines in healthcare is extremely variable ranging from $4.5 \%$ for handwashing guidelines ${ }^{14}$ to $91.6 \%$ for prescribing guidelines in a paediatric ICU. ${ }^{15}$ The adherence rate for this patient acuity tool had been expected to be on the order of $80 \%$, which was in line with internal audits of adherence to the departmental pharmaceutical care guidance. The results from this study may have been due to the fact that the updated guidance had not been fully embedded into practice 6 months after its introduction.

High-risk medications and kidney dysfunction were the most common factors that influenced PAST scoring; no patients were documented as having heart, lung, bone marrow or brain dysfunction. Kidney and liver dysfunction are valid indicators to predict pharmaceutical risk as they have predictable pharmacokinetic effects on medication and can also be easily identified through laboratory biochemistry and haematology testing. ${ }^{16}$ Defining heart, lung and brain dysfunction, as used in the intensive care society's levels of critical care, is more difficult for pharmacists to assess and has less obvious effects on the choice and dosing of medication. Therefore, we believe these indicators may not provide added value to the PAST. Patient factors identified in other research that may improve the ability of PAST to identify high-risk patients without the need for an automated electronic prescribing and administrative system include patient age, number of prescribed medications and difficulty

Table 2 Agreement between expected PAL and pharmacist-documented PAL

\begin{tabular}{llll}
\hline & \multicolumn{3}{l}{ Pharmacist-documented PAL } \\
\cline { 2 - 4 } Expected PAL & Level 1 & Level 2 & Level 3 \\
\hline Level 1 & $4^{*}$ & 6 & 1 \\
Level 2 & 0 & $13^{*}$ & 2 \\
Level 3 & 0 & 6 & $3^{*}$ \\
\hline
\end{tabular}

*Expected and pharmacist-documented PAL match (ie, agreement=0) PAL, patient acuity level. 
Table 3 Pharmaceutical assessment screening tool (PAST) factors for all sampled patients stratified by agreement of pharmacist-documented and expected PALs

\begin{tabular}{|c|c|c|c|c|}
\hline \multirow{2}{*}{$\begin{array}{l}\text { Pharmaceutical assessment } \\
\text { screening factors }\end{array}$} & \multicolumn{4}{|c|}{$\begin{array}{l}\text { Stratified levels of agreement } \\
\text { (number }(\%))^{*}\end{array}$} \\
\hline & -1 & 0 & +1 & +2 \\
\hline $\mathrm{N}$ & $6(100)$ & $20(100)$ & $8(100)$ & $1(100)$ \\
\hline \multicolumn{5}{|l|}{ Organ dysfunction } \\
\hline Kidney & $2(33)$ & $5(25)$ & $1(13)$ & $0(0)$ \\
\hline Liver & $0(0)$ & $1(5)$ & $0(0)$ & $0(0)$ \\
\hline Heart & $0(0)$ & $0(0)$ & $0(0)$ & $0(0)$ \\
\hline Lung & $0(0)$ & $0(0)$ & $0(0)$ & $0(0)$ \\
\hline Bone marrow & $0(0)$ & $0(0)$ & $0(0)$ & $0(0)$ \\
\hline Brain & $0(0)$ & $0(0)$ & $0(0)$ & $0(0)$ \\
\hline \multicolumn{5}{|l|}{ Drugs requiring therapeutic monitoring } \\
\hline Glycopeptide antibiotic & $0(0)$ & $1(5)$ & $0(0)$ & $0(0)$ \\
\hline Aminoglycosides & $2(33)$ & $1(5)$ & $1(13)$ & $0(0)$ \\
\hline Theophylline/aminophylline & $3(50)$ & $0(0)$ & $0(0)$ & $0(0)$ \\
\hline Digoxin & $1(17)$ & $0(0)$ & $0(0)$ & $0(0)$ \\
\hline Carbamazepine & $0(0)$ & $0(0)$ & $0(0)$ & $0(0)$ \\
\hline Lithium & $0(0)$ & $0(0)$ & $0(0)$ & $0(0)$ \\
\hline Ciclosporin & $0(0)$ & $0(0)$ & $0(0)$ & $0(0)$ \\
\hline Amphotericin B & $0(0)$ & $0(0)$ & $0(0)$ & $0(0)$ \\
\hline Triazole antifungals & $0(0)$ & $0(0)$ & $0(0)$ & $0(0)$ \\
\hline \multicolumn{5}{|c|}{ Number of drugs requiring therapeutic monitoring } \\
\hline 0 & $2(33)$ & $18(90)$ & $7(88)$ & $1(100)$ \\
\hline 1 & $2(33)$ & $2(10)$ & $1(13)$ & $0(0)$ \\
\hline 2 & $2(33)$ & $0(0)$ & $0(0)$ & $0(0)$ \\
\hline \multicolumn{5}{|l|}{ Other high-risk medications } \\
\hline Anticoagulation & $1(17)$ & $4(20)$ & $0(0)$ & $0(0)$ \\
\hline Insulin & $4(67)$ & $4(20)$ & $0(0)$ & $0(0)$ \\
\hline Opiates & $1(17)$ & $5(25)$ & $0(0)$ & $0(0)$ \\
\hline Chemotherapy & $0(0)$ & $0(0)$ & $0(0)$ & $0(0)$ \\
\hline Antiretrovirals & $0(0)$ & $0(0)$ & $0(0)$ & $0(0)$ \\
\hline Clozapine & $0(0)$ & $0(0)$ & $0(0)$ & $0(0)$ \\
\hline \multicolumn{5}{|l|}{ Number of high-risk medications $t$} \\
\hline 0 & $0(0)$ & $10(50)$ & $7(88)$ & $1(100)$ \\
\hline 1 & $2(33)$ & $5(25)$ & $1(13)$ & $0(0)$ \\
\hline 2 & $2(33)$ & $5(25)$ & $0(0)$ & $0(0)$ \\
\hline 3 & $2(33)$ & $0(0)$ & $0(0)$ & $0(0)$ \\
\hline ICU step-down patient & $0(0)$ & $1(5)$ & $0(0)$ & $0(0)$ \\
\hline Patient with an infectious disease consult & $0(0)$ & $0(0)$ & $0(0)$ & $0(0)$ \\
\hline Patient with cystic fibrosis & $4(67)$ & $1(5)$ & $0(0)$ & $0(0)$ \\
\hline Organ transplant patient & $0(0)$ & $0(0)$ & $0(0)$ & $0(0)$ \\
\hline Home intravenous therapy & $0(0)$ & $0(0)$ & $0(0)$ & $0(0)$ \\
\hline Out-of-hospital respiratory service & $0(0)$ & $0(0)$ & $0(0)$ & $0(0)$ \\
\hline Patient with HIV & $0(0)$ & $0(0)$ & $0(0)$ & $0(0)$ \\
\hline $\begin{array}{l}\text { Patient with Parkinson's disease on } \\
\text { apomorphine }\end{array}$ & $0(0)$ & $0(0)$ & $0(0)$ & $0(0)$ \\
\hline $\begin{array}{l}\text { High-cost medication (not within NHS } \\
\text { tariff) }\end{array}$ & $0(0)$ & $0(0)$ & $0(0)$ & $0(0)$ \\
\hline $\begin{array}{l}\text { Outside competency of attending } \\
\text { pharmacist } \neq\end{array}$ & $0(0)$ & $0(0)$ & $0(0)$ & $0(0)$ \\
\hline \multicolumn{5}{|l|}{ Pharmacist-documented PAL } \\
\hline 1 & $0(0)$ & $4(20)$ & $0(0)$ & $0(0)$ \\
\hline 2 & $6(100)$ & $13(65)$ & $6(75)$ & $0(0)$ \\
\hline 3 & $0(0)$ & $3(15)$ & $2(25)$ & $1(100)$ \\
\hline
\end{tabular}

${ }^{*}$ Measured agreement of PALs is the difference of pharmacist-documented PAL minus the expected PAL with a range of values from -2 to +2 .

tNumber of high-risk medications includes drugs requiring therapeutic monitoring plus other high-risk medications.

¥0utside competency refers to documentation by a junior pharmacist that the patient's complexity is outside the scope of their competency.

ICU, intensive care unit; NHS, National Health Service; PAL, patient acuity level. communicating with provider (eg, patient and provider speak different languages). 5910

According to the tool, all patients with CF should automatically receive a level-3 designation. However, only one of the five patients in the CF ward had a pharmacist-documented score of 3. In addition, of the six patients with negative PAL agreement (ie, where the pharmacist undervalued patient acuity), four of them were patients located within the CF ward. Since pharmacists working in the CF ward have specialised knowledge in this therapeutic area, they may have adapted the tool to better suit their needs instead of automatically designating all patients as Level 3, which would not assist the individual pharmacists in prioritising patients on that specialist ward.

Variation was also found among six patients with a documented PAL of 2 when the expected level was 1 ; these patients had no high-risk medications and no organ dysfunction. Another patient assigned a Level 3 when the expected level was 1 also had no high-risk medications and no organ impairment. Junior pharmacists within the pharmacy department are encouraged to designate a higher PAL if the patient is outside their personal pharmaceutical competency, which may be the reason for positive PAL agreement (ie, overvaluation of patient acuity) in 9/35 $(26 \%)$ cases. However, this decision would not have been documented anywhere in the clinical notes or prescriptions so could not have been identified during the study.

In total, 15/35 (43\%) patients had pharmacist-documented PALs that did not adhere to pharmacy department PAST guidance. It is thought that possible reasons for this deviation could be that (1) individual pharmacists designate a PAL based on their own interpretation of a patient's pharmaceutical complexity, (2) pharmacists use a higher PAL to serve as a reminder or to prioritise their own work instead of following the departmental PAST guidance and (3) that they do not update the PAL as the patient's condition changes throughout their inpatient stay.

The fact that the tool was not fully validated also means it is not known whether the tool definitely identifies the patients in most need of pharmaceutical care, and it is possible that the clinical experience and judgement of individual pharmacists is important to appropriately assess patient acuity. Additionally, there was a trend where PAL scores were undervalued on wards that typically have more complicated patients (eg, the CF ward), and PAL scores were more likely to be overvalued in wards that typically have less complicated patients. The pharmacists who care for the patients on the CF ward work exclusively with these long-term patients on a regular basis so may be making a relative acuity score for themselves since the tool has no sensitivity in their ward as all patients would have the same PAL.

A qualitative study to identify the attitudes of pharmacists towards the PAST and patient workflow should allow further refinement and ownership of the tool locally. It will also be important to reiterate the true purpose of the tool to the pharmacy team members and to potentially update it by removing unused sections (heart, lung and brain dysfunction) and adding items about the number of medications and patient age.

A major strength of the study was selecting patients within wards by random clusters thus significantly reducing selection bias. Also, while other tools are being developed to measure patient acuity based on pharmaceutical complexity, ${ }^{4-10}$ this study evaluated a tool that can be implemented throughout hospitals where electronic records have not been fully integrated into daily clinical practice.

The main limitations of this study were the small sample size and the use of a non-validated tool which may have led to imprecision in the data. Nonetheless, it adds to the limited 


\section{Key messages}

What is already known on this subject?

- Tools to assess patient acuity by pharmacists are currently being developed in the UK and abroad.

- The purpose of these tools is to reduce the frequency and severity of adverse drug events by ensuring the most pharmaceutically complex patients receive the right level of pharmaceutical care by appropriately experienced pharmacists.

\section{What this study adds?}

- A pharmaceutical assessment screening tool developed to measure patient acuity and help the pharmacy department prioritise pharmaceutical care was only used correctly in approximately half of the patients studied.

- Pharmacists may be using this acuity tool to prioritise their own work schedule, which was not the original purpose of the tool; it is important for pharmacy departments to communicate effectively with pharmacists the true purpose of tools to assess patient acuity.

- Larger scale studies using validated tools in a more diverse patient population are needed across multiple hospital sites to test the generalisability of our findings and before such tools can be reliably used to prioritise pharmaceutical care.

published literature about the current imperfect use of pharmaceutical care priority screening tools in UK hospitals. ${ }^{6-8}$ Larger scale studies using validated tools in a more diverse patient population are needed across multiple hospital sites to test the generalisability of our findings and before such tools can be reliably used to prioritise pharmaceutical care.

\section{CONCLUSION}

A PAST to measure patient acuity and help prioritise pharmaceutical care delivery was used according to departmental guidance in approximately half of the patients studied. Disagreement between pharmacist-documented and expected tool scores needs further investigation to identify reasons for departure from pharmacy department guidance before such a tool can be reliably used to prioritise pharmaceutical care.

\footnotetext{
Acknowledgements RPH travelled to Manchester, UK, as part of an Advanced Pharmacy Practice Experience while a pharmacy student at the University of Kentucky College of Pharmacy in the USA and was supported by the Melody and Stephen J. Ryan Travel Award and the Education Abroad at UK Scholarship sponsored by the University of Kentucky. RPH was the 2015 American Foundation for Pharmaceutical Education (AFPE) Phi Lambda Sigma First Year Graduate School Fellow.
}

Contributors SDW was the consultant pharmacist in medication safety who originally developed the PAST. SDW and DTS made substantial contributions to conception, design and interpretation of data; RPH performed data collection and analyses and drafted the original article. SDW, DTS, RPH and CS all revised the article critically for important intellectual content and provided final approval of the version to be published.

Competing interests SDW is an associate editor for the European Journal of Hospital Pharmacy. No other authors report a competing interest for publication.

Ethics approval Ethics approval was not necessary as this study was deemed a service evaluation project by the hospital's research department.

Provenance and peer review Not commissioned; externally peer reviewed.

\section{REFERENCES}

1 Jennings BM. Patient acuity. In: Hughes RG, ed. Patient safety and quality: an evidence-based handbook for nurses. Rockville, MD: Agency for Healthcare Research and Quality, 2008. http://archive.ahrq.gov/professionals/clinicians-providers/ resources/nursing/resources/nurseshdbk/index.html (accessed Jul 2015).

2 Intensive Care Society. Levels of Critical Care for Adult Patients. Intensive Care Society Standards (revised 2009). London: The Intensive Care Society, 2009. http:// www.ics.ac.uk/ics-homepage/guidelines-and-standards/ (accessed Jul 2015).

3 The Safer Nursing Care Tool (SNCT). The Shelford Group. May 2014. http:// shelfordgroup.org/library/documents/SNCT_A4_23May14a.pdf (accessed Jul 2015).

4 Scoring system could help reduce adverse drug events in hospital patients. University of Florida. 27 Aug 2013. http://news.ufl.edu/archive/2013/08/ scoring-system-could-help-reduce-adverse-drug-events-in-hospital-patients.html (accessed Oct 2014)

5 Falconer N, Nand S, Liow D, et al. Development of an electronic patient prioritization tool for clinical pharmacist interventions. Am J Health Syst Pharm 2014:71:311-20.

6 Cottrell R, Caldwell M, Jardine G. Developing and implementing a pharmacy risk screening tool. Hospital Pharmacy Europe 6 Dec 2013. http://www. hospitalpharmacyeurope.com/featured-articles/ developing-and-implementing-pharmacy-risk-screening-tool (accessed Oct 2014).

7 Mullan N, Jennings A. Pharmacists' Use and Views of the Electronic Prescribing Web Portal [abstract]. GHP/UKCPA Joint National Conference 2013. http://www. ukcpa.net/wp-content/uploads/2014/06/Joint-Conference-2014-Abstracts-only.pdf (accessed Oct 2014).

8 Safadeh M, Pazik L, Kavangh R. A baseline assessment of the pharmaceutical needs of adult patients admitted to Stoke Mandeville Hospital [abstract]. Clin Pharm 2012;(Suppl 2):S36-8. http://www.ukcpa.net/wp-content/uploads/2012/06/ Autumn-Symposium-November-2011.pdf (accessed Jul 2015).

9 Saedder EA, Lisby M, Nielsen LP, et al. Number of drugs most frequently found to be independent risk factors for serious adverse reactions: a systematic literature review. Br J Clin Pharmacol 2015:80:808-17.

10 Urbina 0, Ferrández 0, Grau S, et al. Design of a score to identify hospitalized patients at risk of drug-related problems. Pharmacoepidemiol Drug Saf 2014;23:923-32

11 Cousins DH, Gerrett D, Warner B. A review of medication incidents reported to The National Reporting and Learning System in England and Wales over 6 years (2005-2010). Br J Clin Pharmacol 2012;74:597-604.

12 Blackburn DF, Yakiwchuk EM, Jorgenson DJ, et al. Proposing a redefinition of pharmaceutical care. Ann Pharmacother 2012;46:447-9.

13 Dawson BD, Trapp RG. Basic and clinical biostatistics. 4th edn. New York, NY: Lange Medical Books/McGraw Hill, 2004:118

14 Kim J, Bates DW. Medication administration errors by nurses: adherence to guidelines. J Clin Nurs 2013;22:590-8.

15 Reilly N, O'Neill R, Shetty N, et al. An audit to assess adherence to hospital prescribing guidelines in a tertiary paediatric intensive care unit. Arch Dis Child 2014;99:e3.

16 Buxton ILO, Benet LZ. Pharmacokinetics: the dynamics of drug absorption, distribution, metabolism, and elimination. In: Brunton LL, Chabner BA, Knollmann BC, eds. Goodman \& Gilman's the pharmacological basis of therapeutics. 12th edn. New York, NY: McGraw-Hill, 2011:17-39. 